\title{
Clinical Findings and Management of Pathological Myopic Degeneration with Secondary Choroidal Neo-Vascular Membrane Macular Hemorrhages
}

\author{
Brad Thomas Cunningham* \\ New England College of Optometry, USA
}

Submission: January 29, 2018; Published: February 21, 2018

*Corresponding author: Brad Thomas Cunningham, New England College of Optometry, USA, Email: Bcunning45@gmail.com

\begin{abstract}
Myopia, or nearsightedness affects over 40 percent of the people aged 12-54 in the United States [1]. This gender non-specific condition appears to be increasing [2]. Considered a small subpopulation of myopia, pathological myopia (PM) is a disease affecting up to three percent of the world population with a 31 percent chance of inheritability [3,4]. Since the typical course of PM varies greatly with visual outcomes, it is critical for clinicians to appreciate the severity of the clinical findings, the course of the disease, and take a collaborative approach to treatment options before pursuing interventions. This case report reviews the management of a patient with PM and discusses clinical findings and treatment options as they relate to the case presented. The expected prevalence of myopia and PM demands our attention and further analysis. New and alternative treatment options must be evaluated.
\end{abstract}

Keywords: Pathological myopia; Fluorescein angiography; Choroidal neovascular membrane

\section{Case Report}

Patient \#1, a 36-year-old Filipino female Army active duty dentist, presented in the optometry clinic on May 15, 2012 as an urgent walk-in due to "a black spot" in her vision. She noticed upon looking at her patient through her diagnostic loops, a round spot that she could not see through in the center of her vision in her right eye. She was uncertain how long this spot had been there, but the moment she noticed it she reported to the eye clinic. She was oriented to time, place and person, but demonstrated moderate anxiety.

The patient's last eye exam was overall an unremarkable undilated routine eye exam on a high myope. The patient has an extensive ocular and medical history. She had been diagnosed with pathologic progressive myopia with bilateral staphylomas. She has a history of one small superior temporal retinal hole in the right eye with uncomplicated laser repair at age 18 . The patient had well documented white without pressure temporally and superior lattice degeneration in both eyes. In 2007, she was diagnosed with erythema multiform and was treated for approximately six months with oral prednisone ranging from 5 to $60 \mathrm{mg}$ daily. She has a severe history of chronic ocular and sinus allergies causing bilateral dacryocystitis with multiple dacryocystorhinostomy (DCR) surgeries in 2009. The first round of DCR's were completed by an ear, nose, and throat specialist, but were unsuccessful. She was then referred to an ocular plastic surgeon, who successfully completed bilateral DCR's. The patient healed completely and continues her regiment of daily patanol and artificial tears as needed.

Significant family medical history includes non-hodgkin's lymphoma with her mother, and non-insulin dependent diabetes for both parents and her older brother. Her younger brother was diagnosed with Von-Willebrand's disease shortly after birth.

Uncorrected visual acuity was counting fingers at distance OU and 20/30 at near. The best-corrected visual acuity was similar to her last exam, with a distance manifest refraction of OD -13.00-0.50x170, OS -13.25-0.50x155 providing 20/20-2, $20 / 25+2$, respectively. The patient entered wearing her habitual contact lenses, Acuvue Oasys. The contact prescription of -11.50 / 8.4 / 14.0 OU provided 20/25- OD, OS vision. A slight improvement was observed with an over-refraction of $-0.50 \mathrm{OD}$ and -0.75 OS that provided 20/20- OD, OS vision. Her pupils were equally round and reactive to light with no afferent pupil defect observed OU. Confrontation fields were full to finger count OU. Her extraocular muscles were unrestricted in all gazes and cover test demonstrated orthophoria at distance and 4 prism diopters of exophoria at near. Her intraocular pressures were $12 \mathrm{mmHG}$ OD and $14 \mathrm{mmHG}$ OS using non-contact tonometry. Anterior segment evaluation by slit lamp examination revealed 360 degrees of 0.5-1.0mm limbal neovascularization, slightly denser 
inferiorly OU. In addition, the palpebral conjunctiva revealed multiple scattered retention cysts and concretions with mild giant papillary conjunctivitis OU. She exhibited a healthy and even tear film OU, clear lashes, and enlarged puncta OD>OS due to prior DCR procedures. She exhibited clear center and intact corneas OU; irides were brown OU; anterior chamber appeared clear without cells or flare and the estimate of the anterior chamber was $4 \times 4$ with VonHerrick technique.
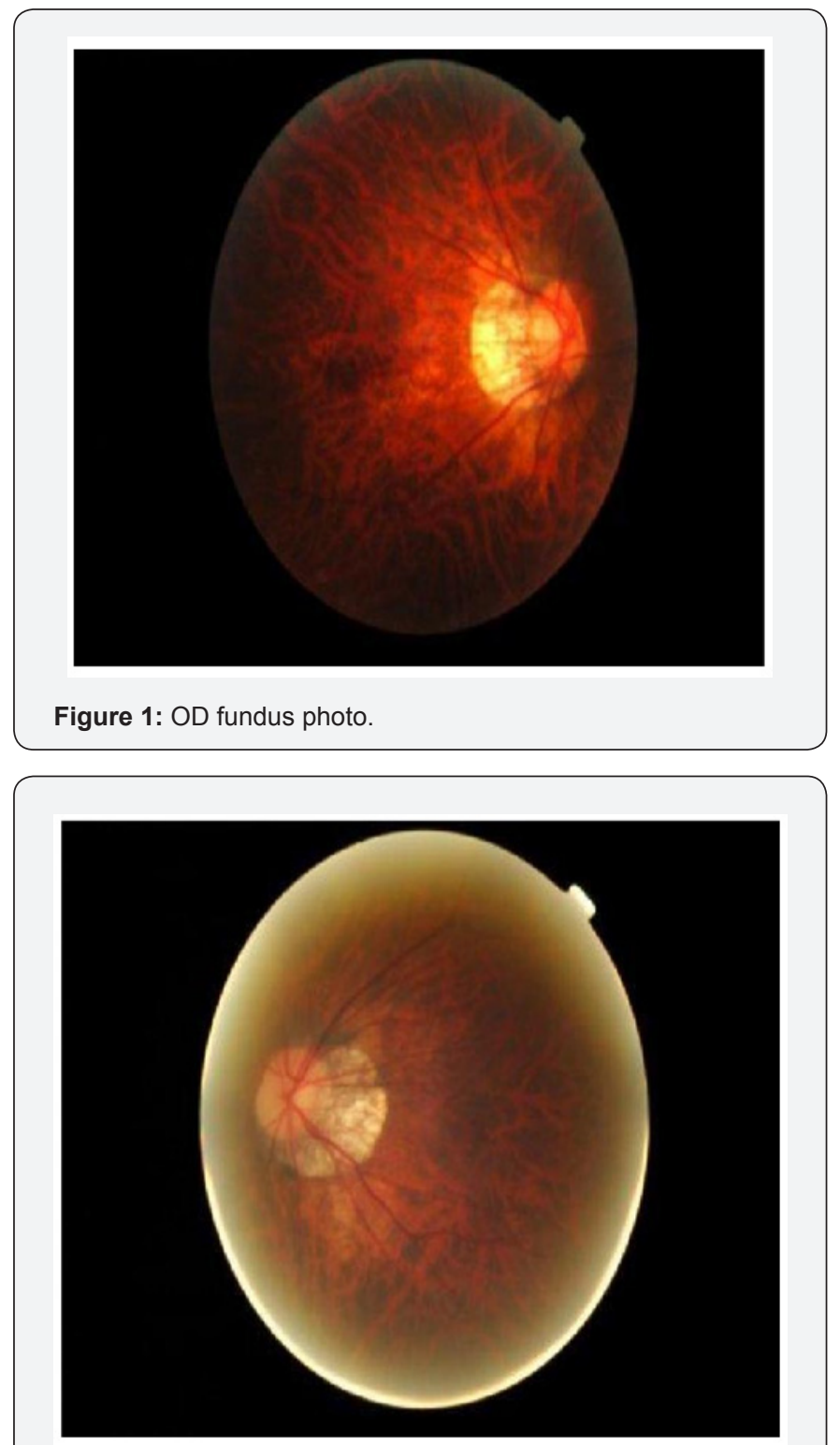

Figure 2: OS fundus photo.

The patient was dilated using one drop of Proparicaine, one drop 1\% Tropicamide and 2.5\% phenylephrine OU. After dilation, an evaluation of the posterior segment by slit lamp with 90D lens and by Binocular Indirect Ophthalmoscope with 20D lens revealed clear lenses OU; slightly tilted myopic optic nerves with 0.30 cup-to-disc ratio $\mathrm{OU}$ with bilateral staphylomas with extensive peripapillary atrophy OU temporally. Extending from the temporal edge of the right staphyloma were small white multifocal lesions to the temporal fovea in a circinate pattern surrounding a $1 / 2$ disc diameter (DD) dark reddish round lesion appearing to be anterior to the macula. No foveal reflex was observed OU and it was difficult to determine if the lesion was raised or sunken. The left macula appeared flat and thin but otherwise within normal limits (WNL). At approximately 10:00 clock hours OD in the peripheral retina, an operculated small round laser treated hole was observed. Otherwise, the tigroid appearing fundus and peripheral retina was flat and intact with no other significant pathology noted OU (Figures $1 \& 2$ ).

The differential diagnoses considered include:

a) Macular hole

b) Macular hemorrhage

c) Myopic macular degeneration

d) Posterior staphyloma progression

e) Pigmented epithelial detachment (PED)

f) Idiopathic central serous chorioretinopathy (ICSC)

i. A macular hole will vary in visual acuity depending on the thickness of the hole. According to Wills Eye Manual, $90 \%$ of macular holes are unilateral and typically present as round, red circular areas in the center of the macula. They are three times more likely in women and usually occurs in sixth to eighth decade [6].

ii. A macular hemorrhage may occur from choroidal or retinal vessel abnormalities. Typically, they result from choroidal neovascular membrane (CNV) secondary to agerelated macular degeneration or other conditions related to CNV like myopia, trauma, ocular histoplasmosis and angioid streaks [6].

iii. Myopic macular degeneration is a generalized diagnosis and disease process that may include a multitude of findings such as lacquer cracks, CNV and macular hemorrhage [6].

iv. Posterior staphylomas are a bilateral presentation with the patient. Continued myopic degeneration may complicate the already thin sclera and enable the aforementioned CNV and/or macular hemorrhage [6].

v. Detachment of the retinal pigmented epithelium, or pigmented epithelial detachment may provide a similar distinct, round appearance but is usually associated with an underlying pathology such as age-related macular degeneration (AMD), CNV or idiopathic central serous chorioretinopathy [6].

vi. Idiopathic central serous chorioretinopathy (ICSC) is a sensory retinal detachment from serous fluid in the macular area due to possible RPE and choriocapillaris dysfunction as well as choroidal ischemia and venous congestion. This 
disease usually affects young men with type-A personalities that are experiencing stressful events [6].

Ocular coherence tomography (OCT) macular thickness studies demonstrated no significant maculopathy OS and an intact, raised, superior right macular lesion. A horizontal line scan (5-line raster) through the lesion showed thin subretinal fluid with no apparent CNV or break in Bruch's membrane. Unfortunately, the distinction of a CNV from the overlying subretinal hemorrhage blurred the view. There was no evidence of a macular hole, PED, or ICSC. Therefore, the patient was diagnosed with a macular hemorrhage and was emergently referred to a retinal specialist for evaluation, fluorescein angiography, and possibly anti-vascular endothelial growth factor (VEGF) treatment.

\section{Retinal Specialty Visit \#1}

Same day evaluation was coordinated with a local retinal specialist who conducted Macula Thickness OCT studies (Figure $3)$.

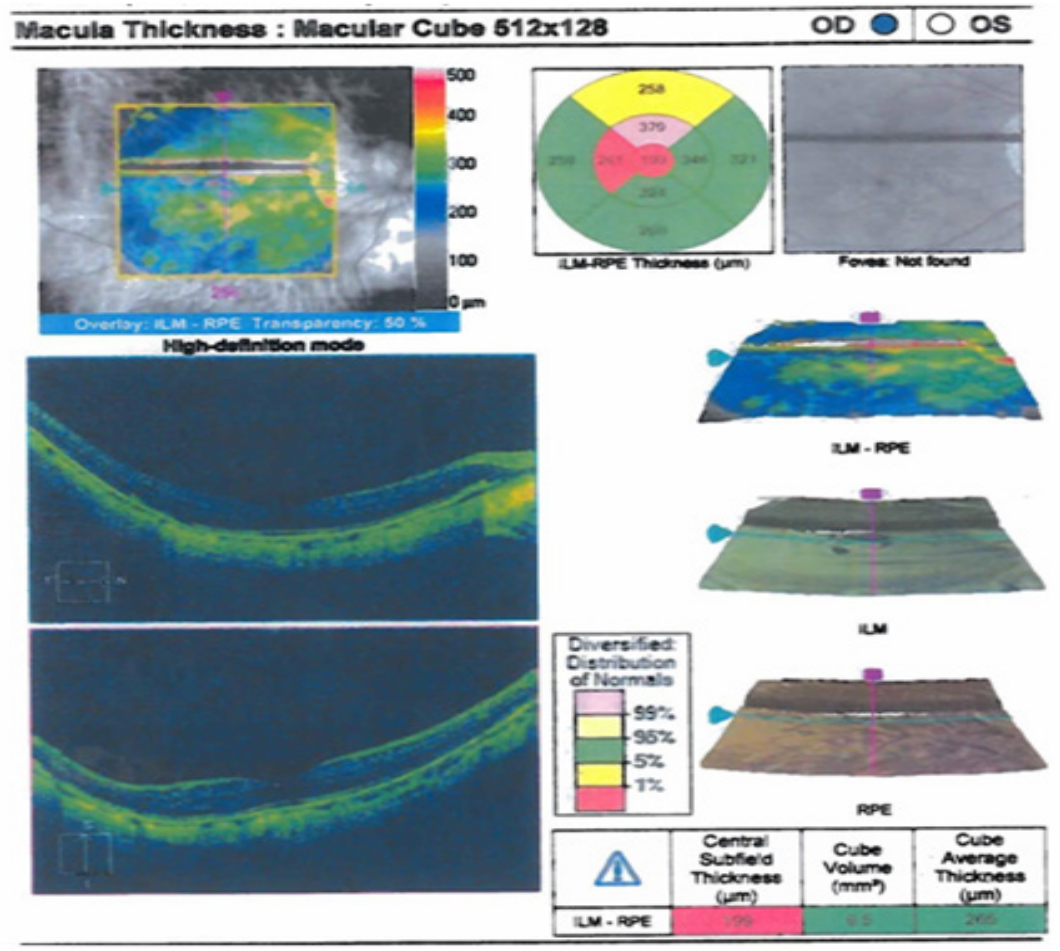

Figure 3: OD Macula Thickness OCT.

According to the study above, there was a large temporal subretinal hemorrhage abutting the temporal edge of the fovea OD only. The OS macula did not exhibit any signs of hemorrhage, leaking, or CNV. The central thickness OD was 316um and OS was $321 \mathrm{um}$. The study appeared well centered and reliable.

Fluorescein Angiography (FA) was also completed and revealed results consistent with subretinal hemorrhage OD. The Cirrus OCT's, FA, and fundus photos demonstrated evidence of treatable subretinal leakage. Although it was not well circumscribed, it is evident that there were abnormal blood vessels under the retina that were leaking. Consequently, intravitreal anti-VEGF therapy was recommended. The patient was advised of the risks associated with intravitreal injections which include, but are not limited to infection, bleeding, and retinal detachment. The medication, Avastin was offered and it was discussed with the patient that although it is considered "off-label" and currently not approved by the FDA for retinal treatment, it has been well studied and approved for treatment of other retinal conditions. She was told of the risk factors to include when given by vein, stroke, hypertension and cardiac complications. She was also told toxicity to the eye is uncertain and long term effects are unknown, and the medication was prepared by a local compounding pharmacy, not by a large pharmaceutical firm. Using a betadine preparation and subconjunctival lidocaine, the right eye was injected with Avastin through the pars plana without any observed complications.

\section{Retinal Specialty Visit \#2}

The patient returned to follow up on the next day on May 16, 2012. She denied any pain or significant change to her vision. She reported the same spot in her right center vision 
but believed it was not as prominent and felt it was slightly diminished. Entering distance acuities with her contact lenses were 20/25+1 OD and 20/20 OS. Amsler grid testing showed a two-square central spot that was faded with a newly noticed red hue OD only. Her intraocular pressure was measured at $12 \mathrm{mmHG}$ OD and OS using non-contact tonometry. Slit lamp examination remained unchanged OU. Mid-dilated fundus assessment remained unchanged OU.

The patient was counseled on the expectations of recovery and positive reinforcement was provided. She was instructed to return to again in four weeks for a follow up and continue to monitor vision at home with Amsler grid testing. She was instructed to return sooner if needed.

\section{Retinal Specialty Visit \#3}

On June 5, 2012, the patient returned for a one-month followup of the right eye subretinal hemorrhage. She complained of a continued spot in the right central vision for approximately one month. No flashes of light, pain, or trauma were noted. Occasional floaters in both eyes were observed. With glasses, her corrected visual acuity was 20/20-2 OD and OS. Her intraocular pressure was measured at $16 \mathrm{mmHG}$ OD and $14 \mathrm{mmHG}$ OS using noncontact tonometry. Her confrontational fields, ocular motility and muscle balance were full OU. Anterior slit lamp examination remained unchanged $\mathrm{OU}$.

The Cirrus macular OCT demonstrated evidence of recovering treatable subretinal OD macular leakage. The central thickness OD was $272 \mathrm{um}$ and OS was $275 \mathrm{um}$. It is evident that there was a break in Bruch's membrane OD with a likely CNV (Figure 4).

Consequently, intravitreal anti-VEGF therapy was recommended again. The patient was advised of the risks associated with intravitreal injections and a second Avastin injection was provided in the right eye only. The left eye appeared to be uninvolved. The patient was counseled on the expectations of recovery and positive reinforcement was provided. She was instructed to return to again in four weeks for follow up and continue to monitor vision at home with Amsler grid testing and may return sooner if needed.

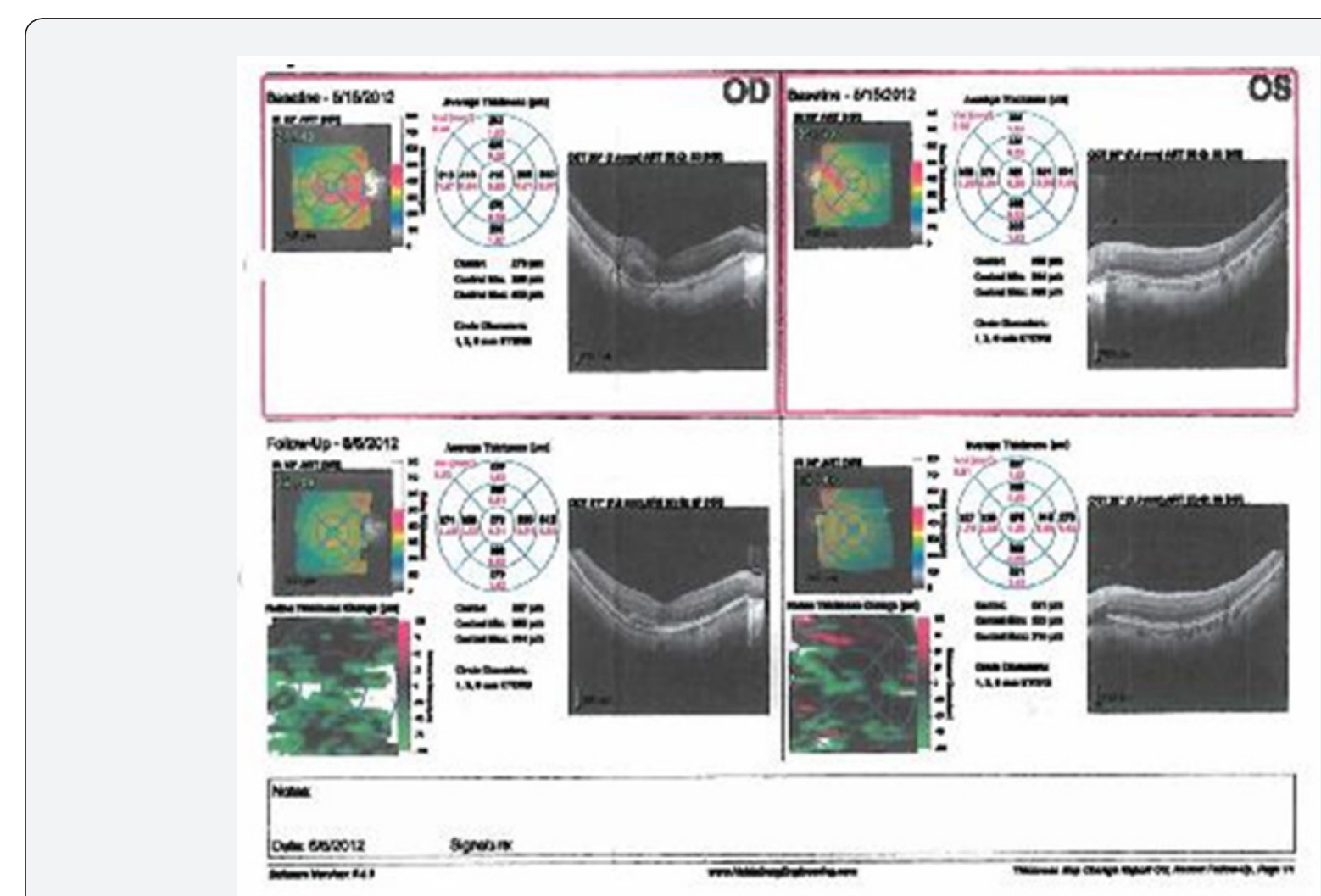

Figure 4: Macular Thickness OCT studies OU.

\section{Retinal Specialty Visit \#4}

The patient returned for a re-evaluation of the right eye subretinal hemorrhage on July 19, 2012. She complained of the continued spot in the right central vision for approximately two months. No flashes of light, pain, or trauma were noted. Occasional floaters in both eyes were observed. With glasses, her corrected visual acuity was 20/20-2 OD and OS. Her intraocular pressure was measured at $17 \mathrm{mmHG}$ OD and 15mmHG OS using non-contact tonometry and blood pressure was measured to be 
114/70. Her confrontational fields, ocular motility and muscle balance were full OU. Anterior slit lamp examination remained unchanged OU.

There was a small area of hemorrhage just abutting the fovea without any significant retinal thickening, intact mid-periphery and in the far periphery OD there is a well-treated retinal break temporally with mature chorioretinal scarring 360 degrees, operculated in nature. Upon examination, the left macula also demonstrated a small subretinal hemorrhage. No peripheral pathology was seen. A small punctate area of hemorrhage just abutting the fovea was observed. The central thickness OD was 379 um and OS was 409um (Figure 5) Comparative analysis was completed and showed a marked improvement of the right macula and a significant worsening of the left macula. The study appeared well centered and reliable.

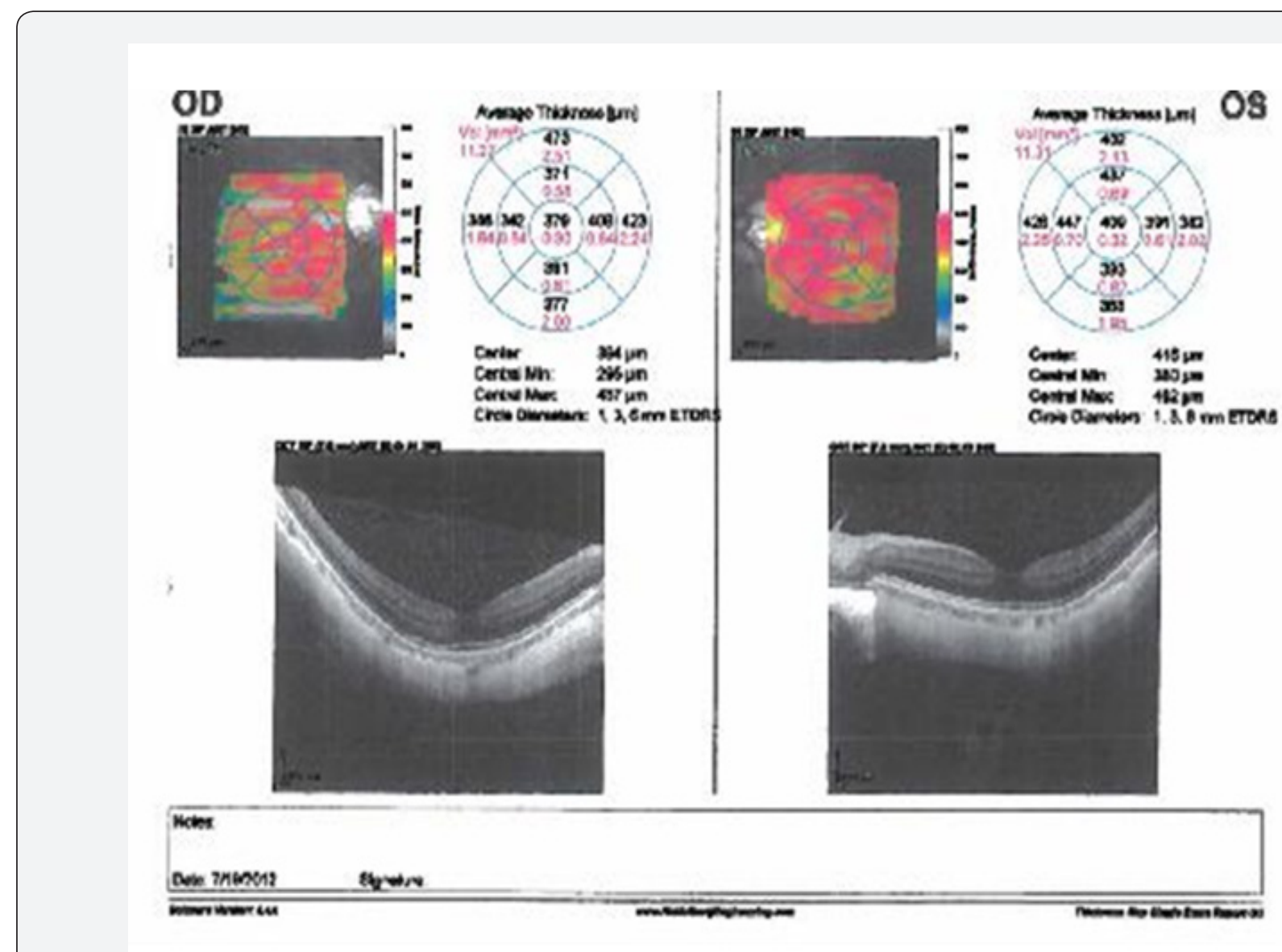

Figure 5: Repeat macular thickness OCT OU studies.

Fluorescein Angiography (FA) was also completed and revealed results consistent with subretinal hemorrhage OU. The FA completed is shown below (Figure 6).

Early phase FA choroidal flush or pre-arterial phase demonstrated thinned RPE and presumed CNV OU caused an early leakage of dye. Both eyes (Figure 6A \& 6G) demonstrate peripapillary absence of pigment epithelium. Later, hypofluorescence (Figure 6B \& 6H) was observed just prior to the arterial phase from the subretinal hemorrhages. During the arterial phase (Figure 6C \& 6I), the hypofluorescence is seen in multiple areas of the right posterior pole and one area in the left macula. Although no neo-vascular network can be clearly distinguished in the FA pictured above, the clinical presentation appeared classically consistent with bilateral CNV secondary to PM.

The Cirrus OCT's and FA demonstrated evidence of treatable subretinal leakage. It is evident that there are abnormal blood vessels under the retina that are leaking OU. Consequently, intravitreal anti-VEGF therapy was completed OU. The patient was advised of the risks associated with intravitreal injections.

The patient was counseled on the expectations of recovery and positive reinforcement was provided. She was instructed to return to again in four weeks for follow up and continue to monitor vision at home with Amsler grid testing and may return sooner if needed. 


\section{JOJ Ophthalmology}


Figure 6: FA OU.

\section{Retinal Specialty Visit \#5}

On August 10, 2012, the patient returned for evaluation of bilateral subretinal hemorrhages from CNV's, likely secondary to underlying myopia. The patient had no entering complaints of spots, flashes of light, pain, or trauma. Occasional floaters in both eyes were observed. With glasses, her corrected visual acuity was 20/20-2 OD and OS. Anterior slit lamp examination remained unchanged $\mathrm{OU}$. The posterior pole of the right eye demonstrated a good foveal depression and no hemorrhage. The left eye also demonstrated resolution of the prior observed small punctate area hemorrhage.

Although excellent treatment response was observed OU, intravitreal Avastin therapy was completed in the right eye only. The patient was counseled on the expectations of recovery and positive reinforcement was provided. She was instructed to return in two months for follow up and continue to monitor vision at home with Amsler grid testing and may return sooner if needed.

Subsequent retinal specialty and optometry follow-ups were completed for the next four years. A compilation of Cirrus OCT measurements is demonstrated below in the discussion.

\section{Discussion}

Pathologic myopia (PM) is a progressive, irreversible disease thought to be related to axial elongation from biomechanical forces of the eye throughout life causing variable weakening and thinning to the retina, choroid, and sclera [1,3]. Although the 
pathogenesis of myopia is still being highly studied, high myopia is exemplified by one's refractive error of at least -6.00 or an axial length of $26.5 \mathrm{~mm}$ or more [3].

The prevalence of PM has been calculated to be at $3 \%$ worldwide with the complication of CNV occurring in approximately $10 \%$ of myopic patients $[3,12]$. PM is one of the leading causes of visual disability in the world from 20-50 years of age [12]. Meta-analysis of the prevalence of myopia predicted $50 \%$ of the world's population will suffer from myopia and $10 \%$ will suffer from high myopia by the year 2050.5

PM has been associated with certain systemic conditions such as Down's Syndrome, Ocular Albinism, Infantile Glaucoma, Marfan's Syndrome, Retinopathy of Prematurity, Ehler's-Danlos Syndrome, low birth weight, and maternal alcoholism $[9,10,12]$. Interestingly, the patient in this case study did not fit into any of these diseases, but did have ocular characteristics. From fundus examination, changes associated with PM include straightened and stretched retinal vessels, temporal peripapillary atrophy (PPA), optic disc tilting, geographic atrophy of the retinal pigment epithelium and choroid, subretinal hemorrhage, CNV, lacquer cracks, and posterior staphylomas [10-12]. Another study has suggested that estrogen may play an important role in CNV development finding the estrogen receptor in 67\% myopic CNV [12]. The patient is female and did have pathognomonic staphylomas, or thinning of the posterior portion of the eye.

Prior to anti-vascular endothelial growth hormone (VEGF) and new treatments, $50 \%$ of patients with staphylomas in both eyes would be legally blind by the age of sixty.10-12 The introduction of anti-VEGF vitreal injections are a game-changer for clinicians to combat PM and wet ARMD complications. It is debatable to discuss this PM case as a variant form of ARMD. Regardless, these two separate diseases have produced the same $\mathrm{CNV}$ result and pose the same risk factors and require the same treatment.

Photo-thermal laser ablation of CNV's used to be the first line treatment. This therapy had a high rate of recurrence and the photo-coagulated retinal scars tended to increase over time and would eventually put the fovea and severely reduced visual acuity at risk [13]. This thermal laser was replaced in the 1990's by photo-dynamic therapy (PDT). The Verteporforin in Photo-dynamic Therapy (VIP) study demonstrated a significant reduction of moderate and severe vision loss with only a $4 \%$ risk of severe vision loss with patients treated with occult CNV [14]. This treatment selectively targets the neo-nets with minimal damage to the retina, RPE and choroid and did not produce the increasing scars produced by thermal laser. Although PDT was better, patients were still being found to have up to $13 \%$ of moderate vision loss and up to $57 \%$ with persistent hemorrhages at one-year post-treatment [14].

Anti-VEGF vitreal injections are now the first line intervention with patients with myopic CNV [13]. When a CNV grows, leaks and bleeds under the retina, a chemical called VEGF is produced to aid in new blood vessel growth. Cancer research has led the way and created anti-VEGF therapies that have proven to be very successful. The FDA has approved many drugs in this class, but three of these medications appear to be most widely used. The top three drugs are tabulated in (Table 1).

Table 1.

\begin{tabular}{|c|c|c|c|c|}
\hline Scientific Name & Trade Name & Company & Cost / Dose & FDA Approval \\
\hline Bevacizumab & Avastin & Genetec & $\sim \$ 50$ & $\begin{array}{c}2004 \text { for certain cancer, not eyes, is } \\
\text { used off-label }\end{array}$ \\
\hline Ranibizumab & Lucentis & Genetec & $\sim \$ 2000$ & 2006 for wet AMD, 2017 for PMD \\
\hline Aflibercept & Eylea & Regeneron & $\sim \$ 1800$ & $\begin{array}{l}2011 \text { for wet AMD, } 2015 \text { for diabetic } \\
\text { macula edema }\end{array}$ \\
\hline
\end{tabular}

The first notable study that found no clinical difference in the effectiveness of these drugs was the Comparison of AMD Treatments Trials (CATT), which was a two-year clinical trial comparing AvastinTM to LucentisTM head to head [15]. Since AvastinTM is one-fortieth the dose provided ocularly, it was believed there may be an increased infection risk due to the potential pharmacist re-dosing procedure; but none was found [15-17]. Studies comparing Avastin, Lucentis, and Eylea have concluded there are minimal differences in risk and effectiveness and the differences appear clinically insignificant [15-20]. These studies have assessed that the frequency of the medication provided is an important consideration. According to the CATT study, approximately ten fewer injections were provided over a two-year period in the as-needed treatment versus the monthly treatment with no clinically significant difference in outcome [21-25]. The amount of appointments, cost, and risk are important considerations of any patient receiving this invasive treatment.

Timely anti-VEGF treatment appears to be critical but some feel it must be used conservatively over time. Longitudinal studies of these treatments are still being conducted but the ones completed have shown very promising results. The MARINA study compared monthly ranabizumab for subfoveal minimally classic or occult with no classic CNV and found $95 \%$ improvement or stabilization of vision compared to $62 \%$ of controls [26]. The ANCHOR study compared monthly ranabizumab to verteporfin PDT for subfoveal classic CNVM and found 95\% of patients treated with ranabizumab had improvement or stabilization of vision compared to $64 \%$ of patient who got PDT [27]. It was also reported $80 \%$ of patients maintained or improved vision at 24 months. Another study grouped 65 patients from 
MARINA, ANCHOR, and HORIZON and evaluated them 7 years after recruitment. They reported $23 \%$ had BCVA of $20 / 40$ or better, $37 \%$ had BCVA of $20 / 200$ or worse, $43 \%$ had unchanged or improved letter score, $68 \%$ had active fluid by OCT, and $98 \%$ had macular atrophy by FAF [28]. This study suggests continued work is needed and provides a bleak outlook for a wet AMD prognosis.

The patient in this case study has been followed for approximately 4 years since the first submacular hemorrhage (Table 2). A new technique in genetic analysis may be able to identify susceptible genes that predispose individuals to myopia in an effort to target and stop myopic progression using gene therapy.29 One study reported the discovery of a chromosomal locus that appears to show a simple autosomal dominant inheritance pattern of high myopia that may further the identification of high myopia development [30].

A relatively new development is the accidental discovery and benefit of using intravitreal gas to displace small submacular hemorrhages in conjunction with tissue plasminogen agent (tPA) to loosen the hemorrhage and physically push the hemorrhage out of the fovea in attempt to maintain baseline visual acuity [31]. Avastin with a tPA injection is also being assessed to reduce the likelihood of future bleeds [31]. For larger subretinal macroaneurysms, vitrectomy with submacular drainage have been assessed and found to have significant complications to include retinal detachments with proliferative vitreoretinopathy [31].

\section{Conclusion}

Although there is no cure for myopia and no cure for progressive pathologic myopia, it is our job and responsibility to do our best to manage the complications associated with it. Proper patient education, early identification and treatment are critical to the prognosis and treatment outcome of CNV. To stabilize the condition and reduce the risk factors for loss of vision is paramount. This chronic and likely progressive condition requires routine exams and a local retinal specialist to provide quick and accessible care when needed.

It is vital to properly assess visual acuity and provide routine dilated fundus evaluations on all patients with high myopia. A detailed macular and peripheral retinal examination are recommended to rule out findings such as lacquer cracks, CNV, myopic retinoschisis, holes, tears, or detachments. Providing education on Amsler grid testing [1] when macular pathology is observed is recommended. Assessment of visual fields [1] and macular thickness OCT studies may be beneficial.

Avastin, as needed, appears the treatment of choice due to the cost, risk, and reduced treatments. Regardless, it is up to the ophthalmologist to assist in making the right treatment choice and there are many therapies to choose from.

The expected prevalence of myopia and PM demands our attention and further analysis. New and alternative treatment options must be evaluated. I will look forward to better treatment options, preventions, or even a cure.

\section{References}

1. Vitale S, Sperduto RD, Ferris FL (2009) Increased prevalence of myopia in the United States between 1971-1972 and 1999-2004. Arch Ophthalmol 127(12): 1632-1639.

2. Williams KM, Bertelsen G, Cumberland P, Wolfram C, Verhoeven VJ, et al. (2015) Increasing prevalence of myopia in Europe and the impact of education. Ophthalmology 122(7): 1489-1497.

3. Stephen Ryan, Andrew Schachat, Charles Wilkinson, David Hinton, SriniVas Sadda, et al. (2013) Retina. ( $5^{\text {th }}$ edn). Elsevier, USA.

4. Tsai MY, Lin LL, Lee V, Chen CJ, Shih YF (2009) Estimation of heritability in myopic twin studies. Jpn J Ophthalmol 53(6): 615.

5. Holden BA, Fricke TR, Wilson DA, Jong M, Naidoo KS, et al. (2016) Global prevalence of myopia and high myopia and temporal trends from 2000 through 2050. Ophthalmology 123(5): 1036-1042.

6. Kunimoto, Derek Y, Kunal D Kanitkar, Mary Makar (2004) The Wills eye manual: office and emergency room diagnosis and treatment of eye disease. Lippincott Williams \& Wilkins, USA.

7. (2016) Lim J (Ed.), Pathologic myopia (myopic degeneration).

8. Wong TY, Ferreira A, Hughes R, Carter G, Mitchell P (2014) Epidemiology and Disease Burden of Patholoic Myopia and Myopic Choroidal Neovascularization: An Evidence Based Systemic Review. Am J Ophthalmol 157(1): 9.e12-25.e12.

9. Callewaert B, Malfait F, Loeys B, De Paepe A (2008) Ehlers-Danlos syndromes and Marfan syndrome. Best Pract Res Clin Rheumatol 22(1): 165-189.

10. Windsor R (2016) Pathological Myopia. Vision Enhancement Magazine.

11. Shih YF, Ho TC, Hsiao CK, Lin LL (2006) Visual outcomes for high myopic patients with or without myopic maculopathy: a 10 year follow up study. Br J Ophthalmol 90(5): 546-550.

12. Pece, Alfredo, Vincenzo Isola, Lucia Vitale (2008) Management of choroidal neovascularization in myopic macular degeneration. Expert Review of Ophthalmology 3(3): 311-323.

13. Adatia FA, Luong M, Munro M, Tufail A (2015) The other CNVM: A review of myopic choroidal neovascularization treatment in the age of anti-vascular endothelial growth factor agents. Surv Ophthalmol 60(3): 204-215.

14.Verteporfin In Photodynamic Therapy Study (2001) Verteporfin therapy of subfoveal choroidal neovascularization in age-related macular degeneration: two-year results of a randomized clinical trial including lesions with occult with no classic choroidal neovascularization-verteporfin in photodynamic therapy report 2 . Am J Ophthalmol 131(5): 541-560.

15. Martin DF, Maguire MG, Fine SL, Ying GS, Jaffe GJ, et al. (2012) Ranibizumab and bevacizumab for treatment of neovascular agerelated macular degeneration: two-year results. Ophthalmology 119(7): 1388-1398.

16. Krebs I, Schmetterer L, Boltz A, Told R, Vécsei-Marlovits V, et al. (2013) A randomised double-masked trial comparing the visual outcome after treatment with ranibizumab or bevacizumab in patients with neovascular age-related macular degeneration. $\mathrm{Br} \mathrm{J}$ Ophthalmol. 97(3): 266-271.

17. Berg K, Pedersen TR, Sandvik L, Bragadóttir R (2015) Comparison of ranibizumab and bevacizumab for neovascular age-related macular degeneration according to LUCAS treat-and-extend protocol. Ophthalmology 122(1): 146-152. 
18. Wells JA, Glassman AR, Ayala AR, Jampol LM, Aiello LP, et al. (2015) Aflibercept, bevacizumab, or ranibizumab for diabetic macular edema. N Engl J Med 2015(372): 1193-1203.

19. Heier JS, Brown DM, Chong V, Korobelnik JF, Kaiser PK, et al. (2012) Intravitreal aflibercept (VEGF trap-eye) in wet age-related macular degeneration. Ophthalmology 119(12): 2537-2548.

20. Browning DJ, Kaiser PK, Rosenfeld PJ, Stewart MW (2018) Aflibercept for age-related macular degeneration: a game-changer or quiet addition?. Am J Ophthalmol 154(2): 222-226.

21. Kook D, Wolf A, Kreutzer T, Neubauer A, Strauss R, et al. (2008) Longterm effect of intravitreal bevacizumab (avastin) in patients with chronic diffuse diabetic macular edema. Retina 28(8): 1053-1060.

22. Neri P, Salvolini S, Mariotti C, Mercanti L, Celani S, et al. (2009) Longterm control of choroidal neovascularisation secondary to angioid streaks treated with intravitreal bevacizumab (Avastin). British Journal of Ophthalmology 93(2): 155-158.

23. Chan WM, Lai TY, Liu DT, Lam DS (2007) Intravitreal bevacizumab (Avastin) for myopic choroidal neovascularization: six-month results of a prospective pilot study. Ophthalmology 114(12): 2190-2196.

24. Peden MC, Suñer IJ, Hammer ME, Grizzard WS (2015) Long-Term Outcomes in Eyes Receiving Fixed-Interval Dosing of Anti-Vascular Endothelial Growth Factor Agents for Wet Age-Related Macular Degeneration. Ophthalmology 122(4): 803-808.
25. Fong AH, Lai TY (2013) Long-term effectiveness of ranibizumab for age-related macular degeneration and diabetic macular edema. Clin Interv Aging 8: 467-483.

26. Kaiser PK, Blodi BA, Shapiro H, Acharya NR, et al. (2007) Angiographic and optical coherence tomographic results of the MARINA study of ranibizumab in neovascular age-related macular degeneration. Ophthalmology 114(10): 1868-1875.

27. Brown DM, Michels M, Kaiser PK, Heier JS, Sy JP, et al. (2009) Ranibizumab versus verteporfin photodynamic therapy for neovascular age-related macular degeneration: two-year results of the ANCHOR study. Ophthalmology 116(1): 57-65.

28. Bhisitkul RB, Desai SJ, Boyer DS, Sadda SR, Zhang K (2016) Fellow eye comparisons for 7-year outcomes in ranibizumab-treated AMD subjects from ANCHOR, MARINA, and HORIZON (SEVEN-UP Study). Ophthalmology 123(6): 1269-1277.

29. Tano Y (2002) Pathologic myopia: where are we now? Am J Ophthalmol 134(5): 645-660.

30. Young TL, Ronan SM, Drahozal LA, Wildenberg SC, Alvear AB, et al. (1998) Evidence that a locus for familial high myopia maps to chromosome 18. Am J Hum Genet 63(1): 109-119.

31. Chen CY, Hooper C, Chiu D, Chamberlain M, Karia N, et al. (2007) Management of submacular hemorrhage with intravitreal injection of tissue plasminogen activator and expansile gas. Retina 27(3): 321-328.

This work is licensed under Creative Commons Attribution 4.0 Licens DOI: 10.19080/JOJO 2018.06.555690 